\title{
Benefícios da fisioterapia pélvica nas disfunções sexuais femininas e na qualidade de vida: revisão integrativa
}

\author{
Benefits of pelvic physiotherapy on female sexual dysfunctions and on quality of life: integrative \\ review
}

\author{
Bruna Padilha Deda ${ }^{1}$, Sthefany Aparecida de Andrade dos Santos ${ }^{2}$, Tania Aparecida Barbosa Rzniski ${ }^{3}$ \\ Centro Universitário Unifacear, Araucária, Paraná, Brasil. *Autor para correspondência. E-mail: saas92@hotmail.com
}

\begin{abstract}
Resumo: Fundamentos: As disfunções sexuais são caracterizadas como qualquer transtorno que trará efeitos e/ou acarretará em um dano na função sexual feminina que $\begin{array}{lll}\text { impacta na qualidade de vida e nas atividades } & \text { diárias. Objetivo: intuito }\end{array}$ desta pesquisa foi realizar uma revisão integrativa com a finalidade de comprovar os benefícios da fisioterapia pélvica no tratamento das disfunções sexuais femininas e na qualidade de vida. Revisão: Trata-se de um estudo da literatura, com busca bibliográfica de artigos científicos publicados plataformas Latino-americano e do Caribe em Ciências da Saúde (LILACS), Medical Literature Analysis and Retrieval System Online (MEDLINE / PUBMED), Scientific Eletronic Library Online (SCIELO), Sciencedirect e Mendeley, nos idiomas inglês e português, no período entre janeiro e junho de 2021. Discussão: Foram encontrados 28.100 títulos, que, após serem filtrados foram reduzidos para apenas 560 artigos. Destes, apenas 7 foram condizentes com a pesquisa. Considerações finais: Baseado nesta pesquisa identificamos que a fisioterapia pélvica desempenha um papel fundamental no tratamento das disfunções sexuais femininas (DSF), e na qualidade de vida, visto que as DSF alteram não somente as estruturas físicas da mulher, mas também aspectos psicológicos e sociais.
\end{abstract}

Palavras-chaves: disfunção sexual feminina, fisioterapia, qualidade de vida.

\begin{abstract}
Background: Sexual dysfunctions are characterized as any disorder that will have effects and / or will cause damage to the female sexual function that directly impact the quality of life and affective food relationships. Objective: The aim of this research was to carry out an integrative review with a view to proving the benefits of pelvic physiotherapy in the treatment of female sexual dysfunction and quality of life. Revision: This is an integrative literature review study, with bibliographic search of published articles published, Latin American and Caribbean platforms in Health Sciences (LILACS), Medical Literature Analysis and Retrieval System Online - (MEDLINE / PUBMED ), Scientific Electronic Library Online (SCIELO), Sciencedirect and Mendeley, combined in English and Portuguese. The search took place between January and June 2021 and the year of publication was not used as a selection criterion. Discussion: The initial search found 28,100 titles, which, after cc, were reduced to just 560 articles. Of these, only 7 were consistent with the survey. Final considerations: Based on this research, it identifies that pelvic physiotherapy plays a key role in the treatment of female sexual dysfunctions (FSD), and in improving the quality of life, since as FSD they change not only the woman's physical structures, but also psychological and social.
\end{abstract}

Keywords: sexual dysfunction, physiotherapy, quality of life.

\section{Introdução}

A sexualidade humana é capaz de influenciar nos mecanismos físicos e psicológicos podendo sofrer alterações multifatoriais (Ferreira, Souza, \& Amorim, 2007). Não pode ser tratada de forma individual, pois é um processo complexo que não se limita aos órgãos sexuais ou ao ato sexual. A organização mundial de saúde (OMS) pontua as disfunções sexuais femininas (DSFs) nas questões de saúde pública (Wolpe et al., 2015). As disfunções sexuais são caracterizadas como qualquer transtorno que trará efeitos e/ou acarretará em um dano na função sexual feminina. Há uma prevalência em mulheres e principalmente nas fases de menopausa e climatério, que são períodos nos quais há desequilíbrio hormonal, sendo capaz gerar dor ou desconforto na relação sexual por falta de lubrificação e por consequência gerar a disfunção (Cavalcanti, 2014). A qualidade de vida por ser um ponto multidimensional, assim como a disfunção sexual, também engloba aspectos físicos, psicológicos, e bem-estar social. A sexualidade e a intimidade são consideradas 
indispensáveis para o bem-estar e, principalmente, para a qualidade de vida. A OMS caracteriza o prazer sexual como essencial para saúde e QV (Tomen, 2015).

Rigodanzo (2008) e Santos et al., (2016), citam que transtornos na sexualidade interferem diretamente na qualidade de vida como a autoestima, segurança, bem-estar, relações interpessoais, confiança da mulher e consequentemente prejudicando a energia primordial instintiva direcionada para o prazer.

São consideradas como as principais disfunções sexuais a dispareunia, vaginismo e anorgasmia devido a frequente incidência de casos. O percentual de mulheres que apresentam algum tipo de disfunção vem aumentando gradativamente, e isso faz com que a situação se torne um problema de saúde pública e muitas dessas mulheres não procuram ajuda médica por receio, vergonha ou por não ter incentivo (Antonioli\& Simões, 2009).

A palavra disfunção refere-se a algo não se comporta da maneira correta, que sugere que há uma anomalia na função. São poucos estudos que tratam dos cuidados de saúde primária ou que sejam relacionados a mulheres saudáveis ou da população em geral (Antônio et al., 2016).

A fisioterapia propõe melhorar a qualidade de vida, oferecendo avaliação, orientações sexuais, esclarecendo dúvidas referente a função sexual, melhora da consciência corporal realizando abordagens comportamentais e programas fisioterapêuticos que buscam apurar uma melhora na sexualidade da mulher (Rigodanzo, 2008).

As técnicas fisioterapêuticas beneficiam em vários aspectos na vida da mulher que sofre com a disfunção sexual, como os exercícios que estabeleçam a contração voluntária do AP agregando vários benefícios como: consciência corporal, aumento da vascularização, aumento da tonicidade e da força da MAP. Sendo assim, é possível manter a cadeia muscular fortalecida, preservando contrastes de problemas físicos futuros (Trindade, 2017).

Segundo Preda \& Moreira (2016), a fisioterapia possui várias técnicas de fortalecimento para a musculatura, porém, para que ocorra o treinamento específico da MAP é necessário realizar sua correta contração. O tratamento fisioterapêutico constitui um avanço recente e demonstra sua eficácia no tratamento das DSF.

Segundo Braz et al. (2009), a cinesioterapia também é uma forma de tratamento de baixo custo e com uma diversidade de exercícios e técnicas. Estudos afirmam que exercícios perineais, equipamentos como cones vaginais, eletroestimulação e sondas de biofeedback também são utilizados como forma de tratamento (Lima \& Freire, 2016; Santos et al., 2016).

São de suma importância novos estudos com ensaios clínicos que apontem resultados através da prática no tratamento das DSF, pois ainda são escassos. Portanto, esta pesquisa teve como objetivo corroborar para o crescimento dos estudos que mostram os benefícios efeitos da fisioterapia pélvica.

\section{Revisão}

Trata-se de uma revisão integrativa caracterizada por meio da busca de artigos científicos baseada nos critérios de inclusão e exclusão, foi processada uma síntese de pesquisa nas plataformas Latinoamericana e do Caribe em Ciências da Saúde (LILACS), Medical Literature Analysis and Retrieval System Online (MEDLINE / PUBMED), Scientific Eletronic Library Online (SCIELO), Sciencedirect e Mendeley, através das palavras chave fisioterapia, disfunção sexual, qualidade de vida.

Foram incluídos ensaios clínicos, no idioma português e inglês, publicados nos anos de 2005 a 2020, que tratassem da fisioterapia na disfunção sexual feminina e os impactos na qualidade de vida. Foram excluídos estudos de revisão, e artigos que não especifiquem o protocolo de atendimento. Os artigos inclusos foram explorados na íntegra e concretizados segundo autor, país, técnicas utilizadas e resultados.

Após serem filtrados conforme as características disponíveis em cada base de dados, teve seu total reduzido para 506 artigos. Abaixo, no quadro 1, estudos foram condizentes com o tema proposto e se enquadraram na metodologia selecionada.

Quadro 1. Apresentação de artigos selecionados.

\begin{tabular}{|c|c|c|}
\hline $\begin{array}{c}\text { Autor/Ano de Publicação e } \\
\text { critérios de seleção }\end{array}$ & Avaliação e intervenção & Resultados \\
\hline $\begin{array}{l}\text { Piassalori, } 2010 \text { Ensaio Clínico: } 45 \text { mulheres } \\
\text { com disfunção } \\
\text { Inclusão: As mulheres selecionadas } \\
\text { apresentavam diagnóstico de disfunção } \\
\text { sexual (transtorno de desejo, excitação, } \\
\text { orgástico e dispareunia). } \\
\text { Exclusão: Mulheres na menopausa ou }\end{array}$ & $\begin{array}{l}\text { As integrantes responderam um questionário de avaliação } \\
\text { da sua função sexual, antes e após o treinamento dos MAP. } \\
\text { O questionário utilizado foi o (FSFI). Ele e composto por } 19 \\
\text { questões, que englobam seis domínios da resposta sexual: } \\
\text { desejo, excitação, lubrificação, orgasmo, satisfação e } \\
\text { dor/desconforto. A pontuação de cada questão é individual, }\end{array}$ & $\begin{array}{l}\text { Os resultados indicam que as mulheres } \\
\text { submetidas ao TMAP apresentaram } \\
\text { melhora significativa nos escores da } \\
\text { função sexual do questionário FSFI (em } \\
\text { todos os domínios e no escore total) e } \\
\text { nas amplitudes da EMG ao longo do } \\
\text { tratamento. Além disso, todas tiveram } \\
\text { aumento da força dos MAP e houve }\end{array}$ \\
\hline
\end{tabular}


climatério, com falência ovariana prematura, grávidas, mulheres com mais do que três gestações e/ou três partos, história pregressa de abuso e/ou estupro, diagnóstico de vaginismo, presença de diabetes, tratamento com ansiolíticos, prolapso de órgãos pélvicos

\section{Janosik, 2005 Amostra: 8 mulheres}

Inclusão: Mulheres com vida sexual ativa há mais de dois anos, que não realizaram qualquer tipo de cirurgia ginecológica, sem disfunções e/ou afecções neurológicas periféricas ou centrais, nuligestas e com o mesmo parceiro no pré e pós-teste. Exclusão: Mulheres que não completaram o programa de utilização do método Pilates.

Costa, 2018.

Amostra: 31 adultas jovens.

Critérios de Seleção: sexo feminino, faixa etária de 19 a 35 anos, heterossexual, ter parceiro fixo, estar com a saúde em dia, não ter tido parto há menos de 6 meses, assinar o termo de consentimento.
Amostra: 10 mulheres

Critérios de seleção: mulheres submetidas a tratamento de câncer de colo de útero e tratamento fisioterapêutico pós- cirúrgico.

Barreto, 2018

Amostra: 34 mulheres

Critérios de Seleção: mulheres com vida sexual ativa, de 20 a

40 anos e que não apresentassem patologias neurológicas. podendo variar de 0 a 5 . Após preencherem o FSFI, as

mulheres foram submetidas a um exame físico, na posição

de decúbito dorsal (quadris e joelhos semifletidos), com

objetivo de avaliar a função dos MAP, por meio da graduação de força muscular e da eletromiografia (EMG). Para avaliar do grau de força, foi utilizado o toque vaginal (bi-digital), considerando os graus de acordo com a escala de Oxford Modificada, que variam de 0 a 5.

Instrumento de avaliação: Ficha de Avaliação adaptada de Etienne e Waitman utilizada para investigar a história ginecológica, a obstétrica e os componentes da resposta sexual feminina.

As mulheres foram submetidas a um programa de Pilates, após assinarem o Termo de Consentimento Livre e Esclarecido (TCLE) e responderem os questionários propostos. O programa contou com uma frequência de duas vezes por semana durante 8 semanas, totalizando 16 sessões com duração de 50 minutos cada uma. Os exercícios foram realizados com auxílio de bola suíça e reformer. $\mathrm{O}$ programa de Pilates constou de 7 exercícios com 12 repetições, conforme a seguinte descrição em cada uma das duas séries previstas: durante a sessão, as mulheres foram orientadas a contrair os músculos do assoalho pélvico no período expiratório, conforme os princípios do método Pilates.

A avaliação do assoalho pélvico se deu através da palpação digital pelo método Perfect e pelos dispositivos biofeedback, Perina e Peritron e como complemento o questionário FSFI (Femeal Sexual Function Index). A avaliação se dividiu em três fases, sendo elas: Fase 1 - antes da intervenção; Fase 2 - 4 semanas após a intervenção; Fase 3 - 8 semanas após a intervenção. Avaliando a função sexual em $\mathrm{p}<0,05$.

melhora das queixas sexuais da maioria delas.

$\mathrm{Na}$ comparação com as avaliações inicial e intermediária, foi observada melhora significativa $(\mathrm{p}<0,0001)$ dos escores do FSFI na avaliação realizada ao final do tratamento. Em relação à EMG, as amplitudes das contrações físicas e tônicas aumentaram significativamente $(\mathrm{p}<$

0,0001 ) ao longo do tratamento. Houve aumento na força do assoalho pélvico, com $69 \%$ das mulheres apresentando, na avaliação final, grau 4 ou 5 e melhora total das queixas sexuais.

As tabelas apresentadas apontam os resultados das três fases de avaliação: Desejo: $4,7 \mathrm{ab} \pm 0,14,6 \mathrm{~b} \pm 0,15,0 \mathrm{a} \pm 0,2$ $\mathrm{p}=0,031$

Excitação: 4,7b $\pm 0,2 \quad 5,0 \mathrm{a} \pm 0,1 \quad 5,0 \mathrm{a} \pm$ $0,1 \mathrm{p}=0,042$.

Lubrificação: 4,6 \pm 0,2 4,9

$0,14,9 \pm 0,2 \mathrm{p}=0,146$.

Satisfação: $5,4 \pm 0,15,1 \pm 0,2$

$5,4 \pm 0,1 \mathrm{p}=0,470$.

Orgasmo: $4,4 \mathrm{~b} \pm 0,24,8 \mathrm{ab} \pm 0,25,0 \mathrm{a} \pm$ $0,2 \mathrm{p}=0,007$.

Dor: $4,5 \pm 0,34,9 \pm 0,25,0 \pm$

$0,2 \mathrm{p}=0,178$

Avaliação da musculatura do assoalho pélvico feito através da escala PERFECT e medido o comprimento vaginal responderam o

questionário Femeal Sexual Function Index

(FSFI).

Foi realizada a desativação de pontos gatilhos, massagem perineal e TMAP com protocolo de exercícios de ganho de força: 3 séries de 10 a 15 contrações voluntárias

máximas sustentadas por 6-8 segundos e com relaxamento por 6-8 segundos; exercícios para ganho de potência: 15 contrações voluntárias

máximas com relaxamentos totais e exercícios para

ganho de resistência: solicitadas contrações voluntárias máximas sustentadas por 30 segundos com relaxamento de 30 segundos até a fadiga. Tratamento realizado uma vez na semana, durante seis semanas.

As avaliadas responderam o questionário Quociente Sexual Versão Feminina (QS - F). A avaliação da função da musculatura do assoalho pélvico se deu pelo instrumento PERINA, em decúbito dorsal, joelhos fletidos e cobertas da cintura para baixo com um lençol. Foi utilizado a escala de 6 força B do aparelho com ajuste de contração de 6 segundos.

A reavaliação foi feita após o término

do tratamento nas 6 semanas. Os dados foram

analisados utilizando $\mathrm{o}$ teste de Wilcoxon.

Para cada ponto de disfunção sexual avaliado, os resultados de antes depois da intervenção fisioterapêutica respectivamente foram: Desejo: 3,6 e 3,6; Excitação: 3,6 e 4,8; Lubrificação: 3,6 e 4,8; Orgasmo: 4,4 e 4,8 ; Satisfação: 4,8 e 4,8; Dor 3,6 e 3,6. Apesar de não significativamente, as mulheres avaliadas e tratadas apresentaram melhora através do tratamento fisioterapêutico.

Através da avaliação para função sexual, observou-se que $3 \%$ das avaliadas (1 mulher) apresentou desempenho desfavorável a regular; $62 \%$ (21 mulheres) obtiveram desempenho de regular a bom; e $35 \%$ (12 mulheres) apresentaram desempenho de bom a excelente.

Os resultados da avaliação da função da força muscular do assoalho pélvico, 9\% da amostra (3 mulheres) classificadas de desfavorável a regular; 53\% (18 mulheres) de regular a bom; e 38\% (13 mulheres) de bom a excelente.

A análise estatística dos dados foi feita através do software SPSS. Comparando os números antes e depois das intervenções fisioterapêuticas obtevese os seguintes resultados para grupo experimental e controle, antes e depois do tratamento, respectivamente: Desejo: 3,59 e 8,38/ 3,56 e 4,00. Excitação: 5,06 e $14,47 / 4,56$ e 5,03 .
A amostra de 64 mulheres foi divida em dois grupos controle e experimental.

Para o grupo Experimental foi designado o tratamento com: palpação digital para avaliar contração e relaxamento, força e resistência avaliada pela escala Oxford e pontos gatilhos avaliados por meio da escala VAS, que mensura intensidade da dor, o questionários FSFI foi utilizado para avaliar as
Inclusão: dor na área genital antes, durante ou após a relação sexual vaginal, que na 8. Exclusão: infecções, tumores, doenças psiquiátricas importantes, vaginismo, vestibulodínia, condições dermatológicas cistite intersticial, endometriose, gravidez, 


\begin{tabular}{|c|c|c|}
\hline $\begin{array}{l}\text { cirurgia nos órgãos pélvicos ou qualquer } \\
\text { tratamento em curso para dispareunia. }\end{array}$ & $\begin{array}{l}\text { fases da resposta sexual. Os avaliados receberam } 10 \text { sessões } \\
\text { de tratamento, sendo } 1 \text { vez por semana durante } 3 \text { meses. Os } \\
\text { métodos utilizados foram liberação de ponto gatilho através } \\
\text { de técnicas manuais, aplicação de TENS com sondas } \\
\text { vaginais, massagem intravaginal profunda e exercícios } \\
\text { graduais para os músculos do assoalho pélvico foram } \\
\text { prescritos para casa, controlados através de uma lista de } \\
\text { avaliação diária. }\end{array}$ & $\begin{array}{l}\text { Lubrificação: } 4,44 \text { e } 14,4 / 5,25 \text { e } 5,94 . \\
\text { Orgasmo:3,63 e } 11,56 / 4,13 \text { e } 4,91 . \\
\text { Satisfação: } 4,31 \text { e } 10,53 / 4,53 \text { e } 4,97 . \\
\text { Sem dor: } 3,88 \text { e } 12,78 / 4,28 \text { e } 4,81 \text {. } \\
\text { FSFI: } 31,16 \text { e } 88,59 / 35,25 \text { e } 38,69 . \\
\text { Força PFM: } 1,72 \text { e } 4,19 / 2,50 \text { e } 2,47 . \\
\text { Resistência PFM: } 4,53 \text { e } 12,25 / 6,44 \text { e } \\
6,56 \text {. VAS: } 9,03 \text { e } 1,41 / 8,34 \text { e } 8,87 . .\end{array}$ \\
\hline $\begin{array}{l}\text { Eftekhar, } 2014 \text { Amostra: } 90 \text { mulheres } \\
\text { Inclusão: Histórico de parto anterior, } \\
\text { disfunção sexual e grau <3 de prolapso de } \\
\text { órgão pélvico. Exclusão: solteiras, doenças } \\
\text { sistêmicas e psicológicas, cirurgia pélvica, } \\
\text { prolapso de alto grau }(3,4) \text {, uso de relaxante } \\
\text { muscular e psicoativo. }\end{array}$ & $\begin{array}{l}\text { A amostra de } 90 \text { pacientes com disfunção sexual e prolapso } \\
\text { dos órgãos pélvicos foi dividia em dois grupos, sendo A } \\
\text { formado por } 45 \text { mulheres a cirurgia de reparo pélvico. E o } \\
\text { grupo B, dividido em } 45 \text { pacientes submetidos a tratamento } \\
\text { fisioterapêutico duas vezes na semana, durante } 8 \text { semanas, } \\
\text { utilizando estimulação elétrica para reconhecimento da } \\
\text { MAP, também foram utilizados biofeedback vaginal e anal, } \\
\text { infravermelho, exercícios de fortalecimento e relaxamento, } \\
\text { incluindo exercícios de Kegel. } \\
\text { A avaliação da função sexual das mulheres avaliadas se deu } \\
\text { através do questionário FSFI. }\end{array}$ & $\begin{array}{l}\text { Dentre as variáveis sexuais avaliadas, no } \\
\text { grupo da intervenção fisioterapêutica } \\
\text { para orgasmo sever, a amostra teve } \\
\text { redução de } 7 \text { para } 1 \text {, após o } \\
\text { tratamento, e } 33 \text { pacientes retornaram a } \\
\text { condição normal. Já o grupo cirúrgico, } \\
\text { teve alteração de } 2 \text { para } 9 \text {. O item } \\
\text { dispareunia, teve os níveis alterados de } \\
13 \text { para } 6 \text {, dessa amostra } 60 \% \text {, } \\
\text { equivalente a } 27 \text { pacientes retornaram } \\
\text { ao estado normal. Já no grupo cirúrgico, } \\
\text { houve alteração de } 32 \text { para } 37 \text { pacientes. } \\
\text { Para libido, os resultados foram de } 25 \\
\text { para } 2 \text { pacientes, após a intervenção } \\
\text { fisioterapêutica. E no grupo cirúrgico foi } \\
\text { de } 20 \text { para } 11 \text {. Excitação no grupo da } \\
\text { fisioterapia foi de } 4 \text { para } 0 \text {, e no grupo } \\
\text { cirúrgico de } 0 \text { para } 1 \text {. }\end{array}$ \\
\hline
\end{tabular}

\section{Discussão}

A busca inicial totalizou em 28.100 títulos, que após serem filtrados conforme os estudos disponíveis em cada plataforma teve seu total reduzido para 506 artigos. Destes, sete estudos condisseram com o tema proposto e se enquadraram na metodologia e com os anos de publicação. As disfunções sexuais feminina são multifatoriais e envolvem aspectos físicos, psicológicos e sociais, ou até mesmo idiopáticas. As principais são: idade acima de 44 anos, menopausa, cirurgias vaginais, fadiga, consumo de álcool e/ou drogas, e desuso da musculatura perineal (Piassarolli, 2010).

A disfunção sexual no Brasil atinge $49 \%$ das mulheres com idade igual ou acima de 18 anos, $67 \%$ das mulheres entre 40 e 65 anos. Aproximadamente um terço das mulheres reclamam de desejo sexual hipoativo, 29,3\% não atingem o orgasmo e 17,8\% sofrem de dispareunia (Cavalcanti, 2014; Abdo \& Fleury, 2006).

Menciona Antônio et al. (2016) o termo disfunção está atrelado à noção de que algo não se comporta como deveria, o que propõe que há um mau funcionamento. Portanto, referente as DSF essa desordem ainda não foi estabelecida. Dos estudos relacionados à disfunção sexual, poucos são os que citam um recurso de tratamento para os cuidados de saúde primária ou aqueles direcionados a mulheres saudáveis ou da população em geral.

Entende-se que a disfunção sexual é como um transtorno transitório ou permanente definido por insatisfação sexual, fazendo com que a esfera motora sofra um bloqueio, afetando uma das fases do ciclo sexual ocasionando grande impacto na qualidade de vida (Souza et al., 2020).

A qualidade de vida por ser uma esfera multidimensional, assim como a disfunção sexual, também engloba aspectos físicos, psicológicos, e bem-estar social. A sexualidade e a intimidade são consideradas indispensáveis para o bem-estar e, principalmente, para a qualidade de vida.

Barreto (2018), destacou em seu estudo que dentro de uma análise são utilizadas amostras de mulheres com a mesma queixa, porém com realidade de vida diferente. Algumas de situação financeira e escolaridades mais altas em relações a outras, estado civil, entre outros aspectos. Esses fatores podem corroborar diretamente nas alterações da função sexual e por decorrência, na qualidade de vida.

Meira et al. (2020) realizou um estudo com o intuito de verificar o que a função sexual afeta em mulheres que estão no período de climatério. Através dos resultados dos questionários aplicados World Health Organization Quality of Life (WHOQOL-BREF) e FSFI, o autor constata que os números apresentados por mulheres que não possuem disfunção sexual são mais altos comparados aos das demais. Dessa forma, é possível identificar que a disfunção sexual atua sobre a função sexual das mulheres.

As disfunções sexuais interferem diretamente no convívio com o parceiro ocasionando ódio por motivos irrelevantes e brigas frequentes, reduzindo o interesse por sexo, perda ou diminuição da motivação pela interação sexual. A falta de compreensão e aceitação do parceiro em relação ao distúrbio contribui de forma 
negativa aos desfechos clínicos do tratamento (Lara et al., 2010). Em estudos feitos por Mendonça e Amaral (2011) mostra que os profissionais que atuam com a sexualidade necessitam compreender a fisiologia e a patologia da disfunção sexual, ou seja, aspectos essenciais para aplicações de condutas de tratamento.

$\mathrm{O}$ tratamento fisioterapêutico constitui um avanço e tem provado seus benefícios diante das DSF. Na literatura encontramos diversos estudos visando à reabilitação do assoalho pélvico através de eletroterapia, biofeedback, terapias manuais e cinesioterapia em geral. O tratamento melhora a sexual, melhora a consciência corporal e sua autoconfiança (Mendonça \& Amaral, 2011).

Com base nisto Pissarolli et al. (2010), desenvolveu uma pesquisa com uma amostra de 45 mulheres, onde responderam o questionário (FSFI), composto por 19 questões que englobou seis domínios da disfunção sexual. A fisioterapia consistiu exercícios em grupo, sendo 10 atendimentos, até duas vezes na semana, por 50 minutos. Os resultados apontaram que as mulheres submetidas aos exercícios obtiveram uma melhora significativa na função sexual. E todas apresentaram aumento de força e melhora nas relações sexuais. Salienta-se que nesse estudo 14 mulheres foram excluídas por não comparecer a primeira avaliação e houve desistência de outras 5. Dessas, 26 participaram do estudo. Dentro dos resultados das mulheres submetidas ao TMAP apresentaram melhora nos escores na função sexual, pelo FSFI e houve melhora na força muscular e nas queixas sexuais da maioria delas. Logo o treinamento dos MAP corrobora de forma positiva nas atividades de vida.

Piassarolli (2010) e Janosik (2005) em seus respectivos estudos, também citam que o TMAP traz resultados positivos em relação a disfunção sexual. Em contrapartida, Batista (2017) afirma que apesar de haver uma correlação entre a força da MAP com a função sexual, a amostra avaliada não constatou resultados estatisticamente satisfatórios, sendo necessário novos estudos para avaliar tais resultados.

Considerando estudo de S. Janosik (2005) foram 8 mulheres participantes, onde foi utilizado como instrumento de pesquisa uma ficha de Etienne e Waitman sendo utilizada para verificar a história ginecológica e a resposta sexual. E FSFI para uma breve avaliação da disfunção sexual da mulher. As selecionadas realizaram Pilates. O programa foi escalado duas vezes por semana durante 8 semanas. Chegando ao total de 16 atendimentos com duração de 50 minutos. Os movimentos foram executados com o uso da bola suíça e reformer. O Pilates constatou sete exercícios com 12 repetições, conforme a descrição prevista em cada uma das séries, na aplicação, as mulheres foram orientadas a contrair os músculos do assoalho pélvico enquanto expiram. Os resultados mostraram uma comparação com a avaliação inicial e intermediaria sendo observada uma melhora significativa nos resultados FSFI na avaliação feita no final do tratamento. Já em relação ao EMG as contrações físicas e tônicas aumentaram ( $p<0,0001)$ ao decorrer do tratamento. Constatou aumento de força do AP com $69 \%$ das mulheres apresentando já na avaliação final grau 4 ou 5 e melhora total das queixas de DSF. Isso mostra que o método Pilates oferece o grau de melhorias dentro da opção de terapia mobilizando os músculos do assoalho pélvico obtendo a melhora na sua função e o aumento de força nos dois tipos de fibras musculares avaliadas.

Bazan e Castro (2016) complementa que a pratica do método tem objetivo de otimizar a força muscular tendo a contração dos músculos diafragma, transverso do abdômen, multifídios e a MAP como centro de força. O Pilates trabalha propriocepção, fortalecimento e a conscientização da musculatura do assoalho pélvico que proporciona uma melhor percepção da região perineal somando para uma melhor satisfação na atividade sexual e a autoimagem da mulher (Brondani, 2014).

Os estudos de Ghaderi (2019) e Pereira (2020) se assemelham por utilizar os métodos de massagem perineal e intravaginal com o intuito de desativar os pontos gatilhos presentes na musculatura do assoalho pélvico (MAP) e promover o relaxamento da região. Além disso, as mulheres avaliadas também receberam prescrição de exercícios para fortalecimento da MAP. Apesar de tratar-se de estudos com amostras distintas, os resultados de ambos foram positivos quanto ao efeito que as técnicas fisioterapêuticas proporcionam.

Ghaderi (2019), mostra em números que a utilização das técnicas manuais de avaliação e terapias manuais como tratamento, associadas a exercícios de fortalecimento e TENS modificou de forma positiva a resposta sexual das mulheres avaliadas, ainda relata que os pontos gatilhos tem influência relevante nas dores durante o ato sexual. Em seu estudo, Pereira (2020) avaliou a função da musculatura do assoalho pélvico pela escala PERFECT, que quantifica a duração da sustentação da musculatura contraída e aplicou o questionário FSFI, que contém 19 questões para avaliar a função sexual de sua amostra. Como forma de tratamento da função sexual e da MAP, alterados devido a um câncer de colo de útero, foi utilizado à liberação de pontos gatilhos através da pressão manual, massagem perineal por deslizamento e exercícios de treinamento dessa musculatura, por sequências de contrações e relaxamentos máximos.

A reavaliação dessa amostra foi feita após o término do tratamento, que durou seis semanas, com total de uma sessão por semana. Os resultados propostos por e Pereira (2020) mostram que apesar de não ser estatisticamente significativos, ainda assim apresentam melhora na estrutura do assoalho pélvico e na função 
sexual decorrentes do CCU. Ainda cita que o treinamento dessas estruturas é fundamental para sustentar os órgãos pélvicos e para a manter a continência urinária e fecal.

Costa (2018) utilizou a palpação digital e o Biofeedback com sonda vaginal para avaliação da MAP, além do questionário FSFI para avaliar a função sexual das mulheres selecionadas na amostra. Prescreveu exercícios de fortalecimento e de conscientização para melhor percepção da musculatura e associação da respiração durante a realização das atividades. Através dos métodos empregados para análise dos resultados, pode-se verificar que houve melhora na função sexual e nas respostas do ciclo sexual. Barreto (2018) em seu estudo com amostra composta por 34 mulheres também utiliza como método de avaliação da força da MAP o dispositivo Biofeedback e associou os resultados da força muscular as respostas obtidas através do questionário Quociente Sexual Versão Feminina (QS - F), que avalia a função sexual das mulheres.

Comparando o grau de satisfação sexual com a força muscular do assoalho pélvico das avaliadas, contatou-se que quanto maior a força, maior é a percepção dessa estrutura e consequentemente maior é a satisfação sexual dos indivíduos (Santos et al., 2016).

No estudo realizado por Efekhar et al. (2014) foi possível verificar os benefícios através tratamento fisioterapêutico pélvico, após o procedimento cirúrgico, nas disfunções sexuais de pacientes com prolapso genital. Foram empregados através da fisioterapia estímulos elétricos, exercícios de fortalecimento, relaxamento e propriocepção da MAP. Os resultados mostraram que a amostra teve queda nos números comparados com os que precedem o tratamento, e muitas das pacientes retornaram ao seu estado normal de função sexual. A intervenção cirúrgica também se mostrou eficaz, porém, em algumas variáveis avaliadas, os resultados foram negativos. Neste caso, portanto, é possível afirmar que a fisioterapia como método de tratamento das DSF é mais satisfatória.

O tratamento fisioterapêutico constitui um avanço e tem se mostrado benéfica no tratamento das disfunções sexuais femininas. Na literatura encontramos diversos estudos visando à reabilitação do assoalho pélvico através de eletroterapia, biofeedback, terapias manuais e cinesioterapia em geral. $\mathrm{O}$ tratamento proporciona para a mulher a melhora de sua saúde sexual, passa a ter maior consciência corporal e melhora sua autoconfiança (Mendonça \& Amaral, 2011).

\section{Considerações finais}

O conhecimento científico relacionado à disfunção sexual apresenta evolução constante, principalmente na área da fisioterapia, o que demonstra um esforço contínuo para entender a complexidade do ciclo sexual, especialmente nas mulheres, a fim de buscar melhora na qualidade de vida.

Com base na análise dos estudos levantados, é possível afirmar que a fisioterapia pélvica desempenha um papel importante no tratamento das disfunções sexuais femininas (DSF), devolvendo a funcionalidade e bemestar das mulheres, através das técnicas de tratamento abordadas.

Considerando a importância da saúde sexual para o bem-estar completo, pesquisas futuras devem ser desenvolvidas com a finalidade de compreender cada vez mais como se comporta o ciclo sexual feminino, e através disso recrutar estratégias de tratamento eficazes, com intuito maior de contribuir com a saúde sexual das mulheres.

\section{Referência}

Abdo, C. H. N., \& Fleury, H. J. 2006. Aspectos diagnósticos e terapêuticos das disfunções sexuais femininas. Revista Psiquiátrica e Clínica, 33, 162-167.

Antônio, J. Z., da Silva, A., da Costa, P. P. B., Jung, D., Pereira, C. F., Nunes, E. F. C., \& Latorre, G. F. S. 2016. Função sexual feminina, desgaste emocional por insatisfação sexual e inteligência emocional. Fisioterapia Brasil, 17(6), 544-550.

Antonioli, R. S., \& Simões, D. 2001. Abordagem fisioterapêutica nas disfunções sexuais femininas. Revista Neurociências, 18, 267-274.

Barreto, K. L. 2018. Treinamento da força muscular do assoalho pélvico e os seus efeitos nas disfunções sexuais femininas. Motricidade, 14, 424- 427.

Batista, N. M. T. L. 2017. Força E Coordenação Motora Da Musculatura Do Assoalho Pélvico E a Função Sexual Feminina. Interdisciplinary Journal of Health Education, 2, 10-15.

Bazan, A. P., \& Castro, G. L. 2016. Prevalência de transtorno orgásmico em mulheres praticantes do método pilates e musculação. Trabalho de Conclusão de Curso. Porto Velho, RO: Centro Universitário São Lucas.

Braz, M. M. 2009. Efeitos da fisioterapia no aprimoramento da vida sexual feminina. Fisioterapia Brasil, 5, 188. 
Brondani, K. H. 2014. Influencia do método pilates sobre a função do assoalho pélvico e sexualidade de mulheres sedentárias. Monografia. Santa Maria, RS: Universidade Federal de Santa Maria.

Cavalcanti, I. F. 2014. Função sexual e fatores associados à disfunção sexual em mulheres no climatério. Revista Brasileira de Ginecologia e Obstetrícia, 36, 97-502.

Costa, C. K. L. 2018. Cuidado fisioterapêutico na função sexual feminina: intervenção educativa na musculatura do assoalho pélvico. Fisioterapia Brasil, 19, 65-71.

Eftekhar, T. 2014. Comparison effect of physiotherapy with surgery on sexual function in patients with pelvic floor disorder: A randomized clinical trial. Iranian Journal of Reproductive Medicine, 12, 7-14.

Ferreira, A. L. C. G., Souza, A. I., \& Amorim, M. M. R. 2007. Prevalência das disfunções sexuais femininas em clínica de planejamento familiar de um hospital escola no Recife, Pernambuco. Revista Brasileira de Saúde Materno Infantil, 7. 143-150.

Ghaderi, F. 2019. Pelvic floor rehabilitation in the treatment of women with dyspareunia: a randomized controlled clinical trial. International Urogynecology Journal, 30, 1849-1855.

Janosik, S. M. 2005. Efeito do Método Pilates Sobre a Função Sexual Feminina. NASPA Journal, 42, 1.

Lara, L. A. S., Silva, A. C. J. S. R., \& Romão, A. P. M. S. 2010. Abordagem das disfunções sexuais femininas. Revista Brasileira de Ginecologia e Obstetricia, 30, 312-21.

Lima, S. L. S. S., \& Freire, L. M. A. B. 2016. Tratamento Fisioterapêutico nos Transtornos Sexuais Dolorosos Femininos: revisão narrativa. Revista Eletrônica da Estácio, 2.

Mendonça, C. R., \& Amaral, W. N. 2011. Tratamento fisioterapêutico das disfunções sexuais femininas - Revisão de Literatura. Femina, 39, 139-142.

Pereira, M. R. L. 2020. Fisioterapia na função sexual e muscular do assoalho pélvico pós-tratamento do câncer de colo do útero. Revista Ciências Em Saúde, 10, 51-55.

Piassaroli, V. P. 2010. Treinamento dos músculos do assoalho pélvico nas disfunções sexuais femininas. Revista Brasileira de Ginecologia e Obstetricia, 32, 234-240.

Preda, A., \& Moreira, S. 2019. Stress urinary incontinence and female sexual dysfunction: The role of pelvic floor rehabilitation. Acta Medica Portuguesa, 32, 721-726.

Rigodanzo, H. (2008). Incidência e abordagem fisioterapêutica nas disfunções sexuais em um grupo de mulheres climatéricas e menopáusicas. Fisioterapia Brasil, 9(6), 392-398.

Santos, J. L., Leão, A. P. F., \& Gardengui, G. 2016. Disfunções sexuais no climatério. Reproducao e Climaterio, 31, 86-92.

Sousa, C. B., de Souza, V. S., \& Figueredo, R. C. (2020). Disfunções sexuais femininas: recursos fisioterapêuticos na anorgasmia feminina pela fraqueza do assoalho pélvico. Multidebates, 4(2), 176-188.

Souza, C. B. 2020. Disfunções sexuais femininas: recursos fisioterapêuticos na anorgasmia feminina pela fraqueza do assoalho pélvico, 176-188.

Tomen, A. 2015. A fisioterapia pélvica no tratamento de mulheres portadoras de vaginismo. Revista de ciências médicas Campinas, 24, 120-130.

Trindade, S. B. 2017. Atuação Do Fisioterapeuta Nas Disfunções Sexuais Femininas. Alumni- Revista Discente da UNIABEU- ISSN, 5, 10-16.

Wolpe, R. H., Toriy, A. M., Silva, F. P., Zom, K., \& Sperandio, F. F. 2015. Atuação da fisioterapia nas disfunções sexuais femininas: uma revisão sistemática. Acta Fisiatr, 22, 87-92.

\section{Minicurrículo}

Bruna Padilha Deda. Fisioterapeuta, Unifacear, Araucária, Paraná, Brasil.

Sthefany Aparecida de Andrade dos Santos. Fisioterapeuta, Unifacear, Araucária, Paraná, Brasil.

Tania Aparecida Barbosa Rzniski. Fisioterapeuta, Unifacear, Araucária, Paraná, Brasil. 
Como citar: Deda, B.P., Santos, S.A.A., \& Rzniski, T.A.B. 2021. Benefícios da fisioterapia pélvica nas disfunções sexuais femininas e na qualidade de vida: revisão integrativa. Pubsaúde, 7, a231. DOI: https://dx.doi.org/10.31533/pubsaude7.a231

Recebido: 22 jul. 2021.

Revisado e aceito: 3 ago. 2021.

Conflito de interesse: os autores declaram, em relação aos produtos e companhias descritos nesse artigo, não ter interesses associativos, comerciais, de propriedade ou financeiros que representem conflito de interesse.

Licenciamento: Este artigo é publicado na modalidade Acesso Aberto sob a licença Creative Commons Atribuição 4.0 (CC-BY 4.0). 